\title{
Space Colonization Using Space-Elevators from Phobos
}

\author{
Leonard M. Weinstein \\ Advanced Measurement and Diagnostics Branch, NASA Langley Research Center, Hampton, VA 23681, USA \\ Phone: (757)864-5543, fax: (757)864-8315, l.m.weinstein@larc.nasa.gov
}

\begin{abstract}
A novel approach is examined for creating an industrial civilization beyond Earth. The approach would take advantage of the unique configuration of Mars and its moon Phobos to make a transportation system capable of raising mass from the surface of Mars to space at a low cost. Mars would be used as the primary location for support personnel and infrastructure. Phobos would be used as a source of raw materials for space-based activity, and as an anchor for tethered carbon-nanotube-based space-elevators. One space-elevator would terminate at the upper edge of Mars' atmosphere. Small craft would be launched from Mars' surface to rendezvous with the moving elevator tip and their payloads detached and raised with solar powered loop elevators to Phobos. Another space-elevator would be extended outward from Phobos to launch craft toward the Earth/Moon system or the asteroid belt. The outward tip would also be used to catch arriving craft. This approach would allow Mars to be colonized, and allow transportation of people and supplies from Mars to support the space industry. In addition, large quantities of material obtained from Phobos could be used to construct space habitats and also supply propellant and material for space industry in the Earth/Moon system as well as around Mars.
\end{abstract}

\section{INTRODUCTION}

Numerous papers and books extensively examine the requirement and the potential value to establish a civilization in space and on other planets. Outstanding examples of discussions that give numerous justifications are found in Stine (1975), O'Neill (1978), Cox (1996), and Zubrin (2000). The most compelling of the arguments to the present author include the following:

i) There is a growing need to obtain new sources of many raw materials for industry. The materials might be obtained from Lunar regolith, from the moons of Mars, and from the asteroid belt.

ii) The problems of increasing energy needs on Earth might be solved by obtaining Helium-3 from Luna or by using large solar power-collecting satellites to beam power to the Earth

iii) Pollution due to industrial activity would be reduced if a significant amount of the industry were conducted in space. This is particularly important if highly polluting or toxic materials are required to be used.

iv) The microgravity and hard vacuum in space might allow many new processes to be done that are not economical or may not even be possible to do on Earth

v) The dispersal of mankind to space or other planets should be done in case critical damage is done to the Earth's biosphere-by an asteroid impact or by accidental or deliberate self-inflicted damage.

vi) Mankind has the desire and drive to expand to any available frontier

Many construction and mining tasks in space, out to Lunar distances, can be done with a combination of preprogrammed and remotely operated (teleoperated) machines. However even in these cases some human presence is required for long term operation. Teleoperation from Earth becomes impractical at distances large enough for a significant time of transit of radio signals to and from Earth, so for these cases the need for a human presence is even more critical.

The expansion of civilization beyond Earth might be done completely without planetary colonization, using large space habitats such as suggested by O'Neill (1978). However the need to obtain and move huge quantities of material to the desired locations, and construction of such huge habitats, is not realistic for the near future. A planet would have much more readily available structural material, and a foundation to build on. The infrastructure to support a space-based industry and civilization could best be located on a planet, and the best candidate for a planet- 
based extension of humankind is Mars. While Lunar and small space-based manned habitats are also likely, the large surface areas and available raw materials on Mars' surface would support the greatest population size. Mars also has several features that are particularly attractive. These include a modest $\mathrm{CO}_{2}$ atmosphere, the presence of large amounts of water at the poles and in the ground, the proximity of the moons Phobos and Deimos, and the proximity to the asteroid belt. Zubrin (1997) gave a detailed discussion of the possible occupation of Mars by humankind.

Mars, like the Earth, has a significant gravity well, and a direct rocket system to go into space from Mars is still very energy-expensive. A rocket lifting from the surface of Mars and going into low orbit requires a velocity change of over $3.6 \mathrm{~km} / \mathrm{s}$. Direct lift from Mars, followed by a trajectory to Earth or other destinations, would require a total of over $6 \mathrm{~km} / \mathrm{s}$ for the elliptical transfer orbit, followed by a deceleration velocity change of about 2 to $3 \mathrm{~km} / \mathrm{s}$ to orbit Luna or the Earth. These missions would require a large amount of fuel and a rocket thrust several times the weight of the fully loaded rocket to take off from Mars.

If a truly economical method of lifting people and material out of the gravity well of Mars were possible, then Mars could support the major portion of space-based activity far more economically than directly from Earth. This is particularly important for extensive industrial capability, which would require a broad base of support facilities. A practical system would also have to have self sufficient life support systems including food production.

The present paper describes a method to lift people and supplies from the surface of Mars with relatively little fuel, and to also boost vehicles and materials on their way toward Earth or the asteroid belt. Large amounts of raw material could be obtained from Phobos and sent to the Earth/Moon system with a far smaller amount of fuel than would be otherwise needed to support space industry.

The only required item needed to accomplish the present proposal that is not currently available is a super high strength cable material. However, there is one candidate, high-strength carbon nanotube cable, which seems to be on the path to practical development. It is anticipated that quantities of this material should be available in just a few decades. The cable strength required to accomplish the present proposal is only a small fraction of the theoretical maximum possible for carbon nanotube cable, so the likelihood of success is reasonably high.

\section{BEANSTALKS AND SKYHOOKS}

In 1960, the Soviet newspaper Komsomolskaya Pravada published an interview with Y. N. Artsutanov which described a means of Earth-to-orbit transportation using a cable system extending from geostationary orbit, and attached to a location on the Earth's equator. A second cable extended outward to keep the center of mass balanced. This concept has been called a beanstalk. The cable would have to extend over $36,000 \mathrm{~km}$ downward. Vehicles could ride up and down the cables to geostationary orbit. If a vehicle went on the outward cable, it could release at greater than orbital velocity. Isaacs, et al. (1966) described several possible uses of such systems throughout the solar system, and gave some design equations. Pearson (1975) analyzed the concept in more detail and gave more extensive derivations for design and structural features of such a structure.

Other approaches were examined, including one by Moravec (1977) in which the center of mass of a multi-cabled structure is placed in a low orbit, and backward rotation of the structure and cables is used to obtain momentary stationary moving tips as they passed near certain locations. This approach was called a skyhook.

The strength to weight of required materials and large mass required to make a beanstalk possible over the Earth may not be practical in the reasonably near future. A skyhook would not require as strong a cable material, but would need a huge anchor mass to not be excessively perturbed from orbit every time an object is lifted. A version of either approach would be possible for Luna, even with present materials. However the mass of material needed for the anchor and cables combined with the fact that only a very low velocity change is needed to land on or escape from the Moon, makes it of limited potential use for that application.

If versions of beanstalks and skyhooks are examined for possible use for Mars, similar problems occur as for the Earth, even though they are somewhat reduced by the lower surface gravity compared to the Earth. However there is a unique variation of the basic concept that has highly desirable features, and that seems possible with materials that 
are currently under development. This unique version takes advantage of the presence of Phobos, the inner moon of Mars, and the rapid development of carbon nanotube materials.

\section{PHOBOS AND THE SPACE-ELEVATORS}

Phobos is in a unique position to assist leaving Mars, and boosting spacecraft on their journey. Phobos is only 6,028 $\mathrm{km}$ above the surface of Mars, which is well below a stationary orbit relative to the ground. It is about $22 \mathrm{~km}$ in diameter, with a mass of about $10^{16} \mathrm{~kg}$. It is thought to contain considerable water, carbon, nitrogen, silicon and several metals, which make it a potential major source of materials for manned space activities. Phobos is also tidallocked to Mars, so that the same face is always pointing to the surface. Phobos orbits Mars with a velocity of about $2.15 \mathrm{~km} / \mathrm{s}$.

Penzo (1984) suggested using tethers on Phobos and Deimos for space-elevators for a Mars transportation system. The version he described would use modest length tethers on Phobos and longer ones on Deimos. The transportation system required full power lift off from Mars and rendezvous with the tethers on both moons in sequence, and would only result in escape velocity from Mars, not elliptical transfer velocity to Earth. The tether system he suggested would help save fuel leaving Mars, but not nearly as much as the present suggested version.

If sufficiently strong carbon nanotube cables could be carried to or manufactured on Phobos, a tethered spaceelevator structure (hereafter called a space-elevator, or elevator) could be fabricated, and attached to Phobos, able to support a significant load all the way to the edge of Mars' atmosphere. Such a space-elevator could also be extended from Phobos outward a comparable distance, and at the outward tip the centrifugal acceleration would significantly exceed the local gravity of Mars. Figure 1 shows a sketch of the basic concept. The orbital velocity along the spaceelevator increases linearly with the radius from the center of Mars. Figure 2 shows how objects would be propelled from Mars to the space-elevator then raised to Phobos. The tip of the elevator, which would be just above the upper edge of the atmosphere, would be moving only about $0.77 \mathrm{~km} / \mathrm{s}$ relative to a non-rotating surface, and the surface of Mars would be moving in the same direction at about $0.25 \mathrm{~km} / \mathrm{s}$ due to planetary rotation. This results in a relative velocity difference of only about $0.52 \mathrm{~km} / \mathrm{s}$ from the ground. The tip passes over a given location about two times per Martian day (which is nearly the same as an Earth day).

The outward space-elevator would be used to catch and release spacecraft for Deimos or to enter and leave the Martian system. The exit direction can be selected by releasing when Phobos is in the desired portion of orbit. The combination of both space-elevators could greatly reduce the thrust level and fuel quantity needed to go from the surface of Mars to Phobos and to arrive at and leave Mars orbit in a selected direction.

The elevators could use solar-cell-powered motors to drive loop lift cables in increments of about $100 \mathrm{~km}$. As the payload is moved higher above Mars, it tries to move laterally from the elevator, since it is moving to an altitude with a different rotational velocity. However, since the payload is attached to the elevator, it gains orbital velocity by the constraining side force imposed by the tether itself. Lifting a payload from Mars to Phobos at an average speed of $133 \mathrm{~km} / \mathrm{hr}$ (arbitrarily selected reasonable speed) would take about two days, so the side force can be considered a very weak acceleration continuously applied over that long time. The side force on the elevator would only be about $5 \mathrm{~N}$ for a $500-\mathrm{kg}$ load, and would be easily constrained.

More than one space-elevator could eventually be built to Phobos. These elevators could be made fully independent, or they could share platforms. This would allow more mass to be carried at a time, and would allow some continuity of activity in case an elevator needed repair.

Phobos is particularly valuable for such a system of space-elevators for several reasons. The first is its huge mass results in any unbalanced forces having small effect on its orbit over long time periods, even with large amounts of lifting. The second is that the materials on Phobos itself can support an extensive space industry. It actually would take less energy and propellant to send material from the outer Phobos elevator to high Lunar orbit than to get it from Lunar material, and incredibly less than to raise it from the surface of the Earth. The combination of location and (probably) available materials make Phobos the logical candidate for a space station and center of space activity, as well as the basis for the space elevator. 


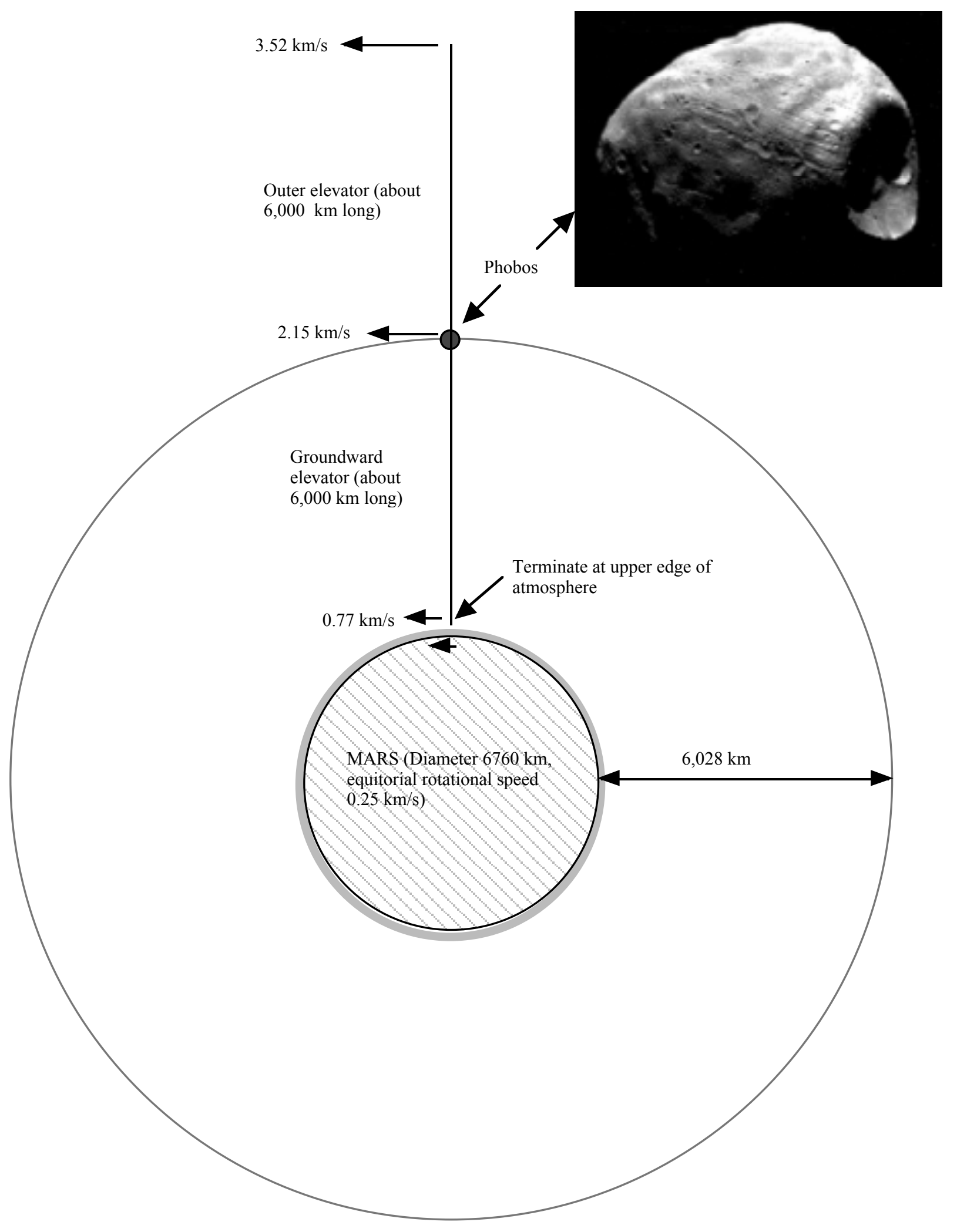

FIGURE 1. Mars, Phobos, and the Space Elevator 


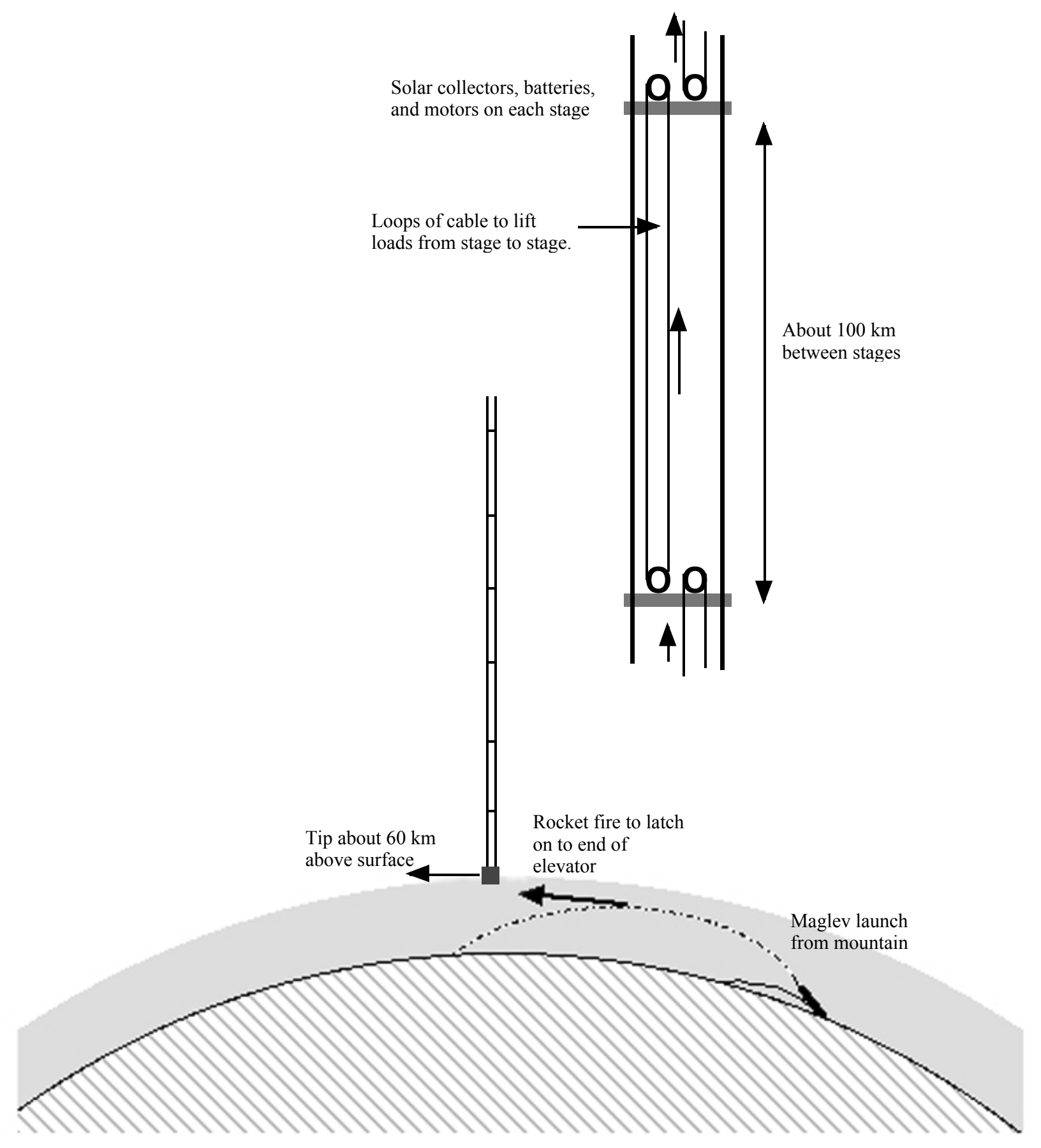

FIGURE 2. Raising Vehicles to Lower End of Elevator

\section{Carbon Nanotube Cables}

Considerable research is currently underway on carbon nanotube material. Walters et al. (1999) describes measurements on small ropes made of single-wall carbon nanotubes that demonstrate that these ropes can have a 
tensile strength over 20 times that of high strength steel. It is not unreasonable to consider that carbon nanotube cable with a reasonable fraction of this strength is likely to be fabricated in quantity within a few decades.

Calculations were made to determine the mass of cable needed for the proposed space-elevators as a function of tensile strength. The cable density was assumed to be similar to that of the carbon nanotubes (about 1.4 grams per cc). The calculations used a maximum loading of about $1 / 3$ the local strength. The cables were made greatly larger than required near the tips to compensate for the smaller cross section being more easily cut by meteoroids. The meteoroid problem and solution will be described later. The required strength of the support cables is not constant at different heights. Due to the large length of the structure, the total mass of the cable itself is generally far greater than the mass of lifted objects. Since the tip only has to support the vehicle load, the maximum vehicle load expected sets the minimum tip strength required. At a location closer to Phobos, the cable has to support the vehicle load and the weight of cable below it. This results in a need for a continually stronger cable all the way to Phobos. Numerical calculation to determine the elevator mass needed were made using 100-km elevator increments, and the cable strength (and thus mass per segment) was adjusted to maintain the required strength to load ratio.

Two local forces act on segments of the elevator when only steady state conditions are considered. These forces are due to Mars' gravity (towards the ground), and the centrifugal force from rotation around Mars (outwards). When the two forces are balanced, the object is in orbit. Equation (1) combines the two effects for the net acceleration along the elevator.

$$
\mathbf{a}_{\text {local }}=\left(4.24 / \mathrm{r}^{2}\right) \times 10^{13}-(5.17 \mathrm{r}) \times 10^{-8} \mathrm{~m} / \mathrm{s}^{2}
$$

where $r$ is the radius from the center of Mars in meters.

The steady state case would result in the elevators moving around Mars while pulled into a straight line pointing from the center of Mars through Phobos. Gravity force, which dominates toward the ground, decreases with increasing altitude until the two forces exactly balance at Phobos. Centrifugal force dominates beyond Phobos, with an increasing force at larger distances.

The calculations were made assuming a vehicle was attached at the lower tip that massed 3,500 $\mathrm{kg}$ (an arbitrarily selected representative mass). A description of one version of a possible vehicle will be discussed later. The solid line on Figure 3 shows the required total mass of an elevator able to hold this load, with a safety factor of 3, for a range of possible cable tensile strengths. The cable mass needed is very large for lower tensile strengths (and would be unrealistic with present materials), but is within the range that seems reasonable for carbon nanotube cable.

The above approach gave the mass for the elevator from Mars to Phobos. The elevator outward from Phobos to release or catch vehicles would have increasing centrifugal accelerations dominating the decreasing Mars gravity. Calculations for this elevator were made assuming a 35,000 kg vehicle at the tip. Lift stages massing $1,000 \mathrm{~kg}$ and lifted masses (per stage) of 5,000 kg are assumed for this case. The larger mass is needed since interplanetary missions would require a larger vehicle than transit from Mars to Phobos. Spacecraft could dock at the tip, and small modules could be detached to rapidly carry people and supplies to Phobos and back. The dashed line on Figure 3 shows the total mass of this elevator as a function of tensile strength needed for this type of operation. The structure mass for the outward elevator is smaller at lower tensile strengths than that for the elevator up from Mars even though the supported mass is 10 times as large, due to the lower maximum acceleration value on the outward leg elevator. Both required elevator masses become smaller with very high tensile strength cables. The curves cross with increasing tensile strengths, and the downward leg become quite small for the higher tensile strengths.

\section{Vehicles and Operations}

Since raw materials would be readily available from Phobos (and Deimos), and since solar energy is reasonably available even to the distance of Mars, a major portion of industrial activity would likely be done in space, or even directly on Phobos. The activity on the surface of Mars might be very extensive, with a large population present, but much of the activity would be done for use on the planet. The main items to be transported from the ground to Phobos might include people, food supplies, and high tech products that required an extensive support infrastructure to fabricate. This suggests that only modest lift capability might generally be needed per trip, although frequent lifts 


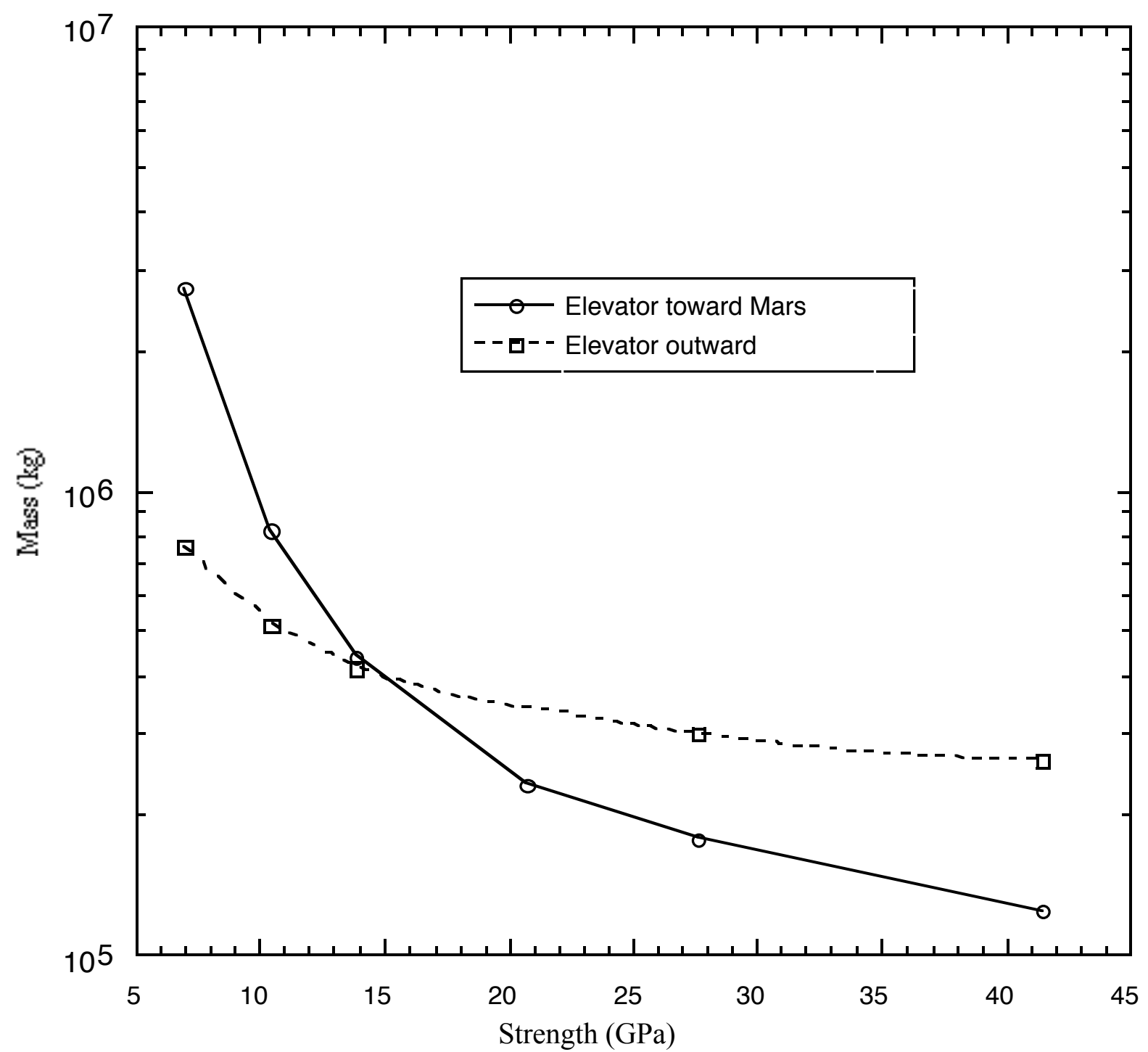

FIGURE 3. Mass of Space Elevators as Function of Strength. A safety factor of 3 was used

may be needed. This modest lift requirement is the basis for the approach chosen for the present paper. Since the elevator solar cells, motors, etc. required to lift items at reasonable speeds needed to be fairly small, a nominal package mass per loop was selected to be about $500 \mathrm{~kg}$. Several packages this size (including people carriers) could be carried to the elevator tip in one flight vehicle, and placed on the lift loops one at a time. A total mass for the vehicle of 3,500 kg, including payload and some fuel margin, seems reasonable for this approach, with up to 4 modules carried in a trip. Only one module would be attached on a lift loop at a time, then the carrier vehicle (empty mass about $1,500 \mathrm{~kg}$ ) would detach and return to the ground with a large paraglider for reuse. In addition to the support cables and lift loops, there would be additional mass due to cable lift stages located every $100-\mathrm{km}$, and lift equipment. The lift stages would include solar cells, motors, pulleys, and structure, and also small batteries for continuous power even during times when Mars occults the sun. Since Phobos circles a particular ground location on Mars about two times a day, two vehicles loads could be launched a day from one location, and even more if several locations were used. In addition, more than one elevator to Phobos could be used to further increase lift capacity. The elevator tip could also be used to rapidly transport payloads around the planet.

The basic lift vehicle could consist of a Maglev boosted rocket powered vehicle with a maximum thrust slightly higher than the fully loaded vehicle weight on Mars. An engine thrust of about 15,000 N would be adequate. The 
vehicle would be launched from a track running up a mountainside and directed in the path of the elevator. A launch velocity of about $1 \mathrm{~km} / \mathrm{s}$ at an elevation of about 40 degrees would be sufficient to approach the tip of the elevator at nearly the correct velocity. The rocket would then be used to dock with the elevator tip. The lift packages would be attached to the loops one at a time, with enough time between to carry each packet to the next loop. The empty vehicle would then detach in time to return back to the launch site and deploy a decelerator chute. When the speed and altitude desired is reached a large but lightweight parasail could be deployed for a soft landing. The vehicle could be either fully automatic, or use teleoperation control. The vehicle could be reused many times, resulting in relatively low cost operation.

Unoccupied lift modules could be small and compact with a very lightweight structure. Modules to carry people might be 2 to $2.5 \mathrm{~m}$ diameter and hold 2 to 4 people each. The lift trip to Phobos would take about 2 days, so air, supplies and a reasonable comfortable module would be required.

Going from Phobos (or directly from space) to the surface of Mars would not require using the elevator. A vehicle could be Maglev launched from Phobos in the direction opposite orbital motion, with the new orbit cutting into Mars' atmosphere. A combination of aerobreaking and use of a large paraglider could then allow a soft landing. A direct landing from space might require more aerobreaking, but would otherwise be similar.

The elevator tip outward from Phobos might be made into a docking station for spaceships to land on. The people and supplies could again be carried in separate modules to allow easy loading and unloading of the ships. The tip velocity at $6,000 \mathrm{~km}$ out would be $3.6 \mathrm{~km} / \mathrm{s}$, and objects released from the tip would retain a hyperbolic velocity (relative to Mars) of $2.6 \mathrm{~km} / \mathrm{sec}$, which is the value need for a Hohmann ellipse transfer to Earth. It is also a significant fraction of the mission velocity needed to go to the inner portion of the asteroid belt. The boost from this release (or capture) would greatly reduce the mission delta velocity to go to (or from) Earth or the Asteroid belt, and thus greatly reduce the propellant required.

\section{The Problem with Meteoroids, and How To Minimize It}

Long cables used in space have a potential problem with meteoroid damage. While a cable may have a fairly small diameter, the large length still results in a large cross section, and thus high probability of being cut. If full-length cables $(6,000 \mathrm{~km}$ long) were used for the Phobos elevators, they would not last long enough to be practical. Even running cables only $100 \mathrm{~km}$ between stages and attaching them to the stages, would result in too limited a usable lifetime. Using larger numbers of smaller separate cables allows some cable strands to fail, and still allow the overall cable to be usable. However, since smaller particles can now cut the cable strands, and the effective capture-area increases, then total failure still occurs rapidly, and may be about as bad. The most practical solution, described in a paper by Foreward (1992), is to make a net of fairly small cable strands, with full strength joints between strands at fairly short strand lengths. The strands need to be held apart so that a meteoroid cutting one strand would not have a high chance of cutting several nearby. The resulting cable would look like a long skinny fish net. Estimations of damage rates have been made using meteoroid flux vs. diameter data from Grun et al. (1985), and assuming that a meteoroid can significantly degrade or even cut a strand if it is over 0.05 times the strand diameter. Even though large numbers of strands along the net would fail per year, the redundancy of tension paths would allow the system to be safe for decades. If repairs were made every few years, the system could be made reliable for a long period of time.

Meteoroids would pose a much smaller but not insignificant problem to items being raised from Mars to Phobos, or from Phobos to the outer tip. Since the trip time is only a couple of days, and the area of an object is only a few square meters, the probability of impact by large enough meteoroids to puncture a vehicle is not significant for reasonably thick vehicle skins. It is desirable that the required thickness be small as possible so that structural mass is minimized, to allow more payload. Use of a double or multiple wall structure as described by Cour-Palais (1990) has been shown to give more protection with less mass than thicker single walls. For example, if the outer wall of a double walled skin was about 0.2 times the thickness of the inner wall, the resulting structure would have a comparable level of penetration blockage as a single wall 10 times as thick as a the total for the double wall. 


\section{SUMMARY}

A novel approach is examined for creating an industrial civilization beyond the Earth. The approach would take advantage of the unique configuration of Mars and its moon Phobos to make a transportation system capable of lifting frequent payloads from the surface of Mars to space and accomplishing this at a low cost. Mars would be used as the primary location for support personnel and infrastructure. Phobos would be used as a source of raw materials for space-based activity, and as an anchor for tethered carbon-nanotube-based space-elevators to help raise people and payloads from Mars to space. One space-elevator would terminate at the upper edge of Mars' atmosphere $(6,000 \mathrm{~km}$ long). This terminus would only be moving about $0.52 \mathrm{~km} / \mathrm{s}$ relative to the surface. Small craft could be launched from Mars' surface at a modest velocity and small rockets used to rendezvous with and attach the craft to the moving elevator tip. Staged cable lifts could then raise modules from the craft to Phobos, then the empty craft detached and landed with a paraglider. Landing on Mars from space could be done directly with a combination of aerobreaking and use of a large paraglider to land.

Another space-elevator would be extended outward of Phobos an additional 6,000 km to launch craft toward the Earth/Moon system or the asteroid belt. Release at the outward elevator tip velocity of $3.52 \mathrm{~km} / \mathrm{sec}$ would result in a hyperbolic velocity of about $2.6 \mathrm{~km} / \mathrm{sec}$. This is the Hohmann elliptical transfer velocity needed to reach the Earth/Moon system, and is also nearly the transfer velocity needed to reach the inner edge of the asteroid belt. This velocity boost would considerably reduce onboard propellant needs for space transportation. This outward elevator tip could also be used to catch arriving craft, with staged elevators also bringing the vehicles or carrier modules from the vehicles to Phobos.

These space-elevators would allow low cost movement of people and supplies from Mars to Phobos and from Phobos to interplanetary space. This approach would allow Mars to be used to support an extensive space industry. In addition, large quantities of material obtained from Phobos could be used to construct space habitats and also supply propellant and material for space industry in the Earth/Moon system as well as on and around Mars.

\section{REFERENCES}

Cour-Palais, and Crews, J.L., International Journal of Impact Engineering, 10, 135-146, 1990.

Cox, D.,and Chestek, J., Doomsday Asteroid: Can We Survive? Prometheus Books, Amherst, N.Y., 1996.

Foreward, R.L., "Failsafe Multistrand Tether Structures for Space Propulsion," in AIAA/SAE/ASME/ASEE $28^{\text {th }}$ Joint Propulsion Conference, AIAA Paper 92-3214, Nashville, Tennessee, 1992.

Grun, E., Zook, H.A., Sectig, H., and Giese, R.H., Icarus, International Journal of Solar System Studies, 62, 244-272, 1985.

Isaacs, J.D., Vine, A.C., Bradner, H., and Bachus, H., Science, 151, 682-683, 1966.

Moravec, H., Journal of the Astronautical Sciences XXV, 4, 307-322, 1977.

O'Neill, G.K., The High Frontier, Bantam Books, New York, 1978.

Pearson, J., Acta Astronautica, 2, 785-799, 1975.

Penzo, P.A., AAS, Science and Technology Series, 62, 445-465, 1984

Stine, G.H., The Third Industrial Revolution, G. P. Putnam's Sons, New York, 1975.

Walters, D.A., Erickson, L.M., Casavant, M.J., Liu, J., Colbert, D.T., Smith, K.A., and Smalley, R.E., Applied Physics Letters, 74 No. 25, 3803-3805, 1999.

Zubrin, R. with Wagner, R., The Case For Mars: The Plan to Settle the Red Planet and Why We Must, Simon and Schuster, New York, 1997.

Zubrin, R., Entering Space, Jeremy P. Tacher/Putnam, New York, 2000. 